\title{
A Generalized Markov Chain Model for Effective Analysis of Slotted IEEE 802.15.4
}

\author{
Pangun Park, Piergiuseppe Di Marco, Pablo Soldati, Carlo Fischione, Karl Henrik Johansson
}

\begin{abstract}
A generalized analysis of the IEEE 802.15.4 medium access control (MAC) protocol in terms of reliability, delay and energy consumption is presented. The IEEE 802.15.4 exponential backoff process is modeled through a Markov chain taking into account retry limits, acknowledgements, and unsaturated traffic. Simple and effective approximations of the reliability, delay and energy consumption under low traffic regime are proposed. It is demonstrated that the delay distribution of IEEE 802.15.4 depends mainly on MAC parameters and collision probability. In addition, the impact of MAC parameters on the performance metrics is analyzed. The analysis is more general and gives more accurate results than existing methods in the literature. Monte Carlo simulations confirm that the proposed approximations offer a satisfactory accuracy.
\end{abstract}

Keywords: IEEE 802.15.4 standard, Markov chain model, Retry limits and acknowledgement, Model approximation.

\section{INTRODUCTION}

Wireless Sensor Networks (WSNs) have revolutionized the world of distributed systems and have enabled several new applications. The IEEE 802.15.4 standard has received considerable attention in academy and industry as a possible low data rate and low power protocol for WSNs [1]. Understanding reliability, delay and energy consumption of IEEE 802.15.4 networks is essential to characterize the fundamental limitations of this protocol and optimize its parameters.

Several simulations-based studies e.g., [2]-[4], as well as more recent analytical works, e.g., [5]-[8], investigate the delay, throughput, and energy consumption of IEEE 802.15.4. Most of the theoretical studies are based on the Markov model initially proposed by Bianchi [9] for the IEEE 802.11 standard [10]. The model describes the basic functionalities of IEEE 802.11 through a Markov chain under saturated traffic and ideal channel conditions. Extensions of this model have been used to analyze the packet reception rate [11], the delay [12], [13], the medium access control (MAC) layer service time [14], [15] and throughput [16], [17] of IEEE 802.11. A simple and effective analysis of delay distribution is studied for IEEE 802.11 in [18].

Inspired by Bianchi's work, a Markov model for IEEE 802.15.4 and an extension with acknowledgement mechanism have been proposed in [5] and [8], respectively. A modified Markov model including retransmissions with finite retry limit

The authors are with the ACCESS Linnaeus Center, Electrical Engineering, Royal Institute of Technology, Stockholm, Sweden. E-mails: \{pgpark, pidm, soldati, carlofi,kallej\}@ee.kth.se.

This work was supported by the EU project FeedNetBack, the Swedish Research Council, the Swedish Strategic Research Foundation, and the Swedish Governmental Agency for Innovation System.

978-1-4244-5113-5/09/\$25.00 (C)2009 IEEE has been studied in [7] for the slotted carrier sense multiple access with collision avoidance (CSMA/CA) mechanism of IEEE 802.15.4. However, the energy consumption and throughput analysis under unsaturated traffic show a weak matching with the simulation results.

In this paper we present an accurate model and analysis of IEEE 802.15.4 MAC protocol in terms of reliability, delay and energy consumption. Unlike previous related works, we propose a generalized Markov model of the exponential backoff process with retry limits and acknowledgements under unsaturated traffic regime. We show that our Markov chain gives an accurate model of the reliability, delay and energy consumption of IEEE 802.15.4. Evaluating these performance metrics asks in general for heavy computations. As such, these expressions may not be directly applied to optimize the IEEE 802.15.4 MAC parameters by an in-network processing of the nodes [19] since complex computations are out of reach for today's sensing devices. To overcome this problem, we devise a simplified and effective method that drastically reduces the computation complexity while ensuring a satisfactory accuracy. Furthermore, we use these results to analyze the performance of IEEE 802.15.4 as functions of the MAC parameters and collision probability. Monte Carlo simulations confirm the validity of our analysis.

The remainder of this paper is as follows. In Section II, we describe the slotted CSMA/CA mechanism of IEEE 802.15.4 standard. We propose a Markov chain model of CSMA/CA with retry limits and unsaturated traffic in Section III. In Section IV we present an accurate analysis of the reliability, delay and energy consumption. Then, in Section V an approximated analysis is developed. In Section VI, we validate our analysis by Monte Carlo simulations. Section VII concludes the paper.

\section{OVERVIEW OF THE IEEE 802.15.4}

The IEEE 802.15.4 standard specifies MAC and physical (PHY) layers. The CSMA/CA is used along with a slotted Binary Exponential Backoff (BEB) scheme to reduce collisions due to simultaneous node transmissions. The standard defines two channel access modalities: the Beacon-enabled modality, which uses a slotted CSMA/CA and exponential backoff, and a simpler unslotted CSMA/CA without beacons.

Consider a node trying to transmit. In slotted CSMA/CA of IEEE 802.15.4, first the MAC sub-layer initializes four variables, i.e., the number of backoffs $(\mathrm{NB}=0)$, contention window $(\mathrm{CW}=2)$, backoff exponent $(\mathrm{BE}=$ macMinBE $)$ and retransmission times $(\mathrm{RT}=0)$. Then the MAC sub-layer delays for a random number of complete backoff periods in 


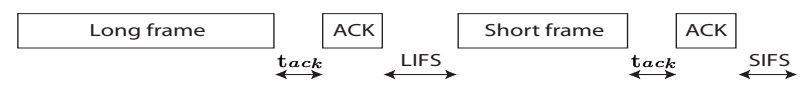

(a) Acknowledged transmission

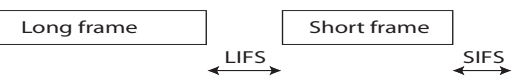

(b) Unacknowledged transmission

Fig. 1. IFS data transmission mechanism with and without acknowledgement.

the range $\left[0,2^{\mathrm{BE}}-1\right]$ units. When the backoff period is zero, the node performs the first clear channel assessment (CCA). If two consecutive CCAs are idle, then the node commences the packet transmission. If either of the CCA fails due to busy channel, MAC sublayer will increase the value of both $\mathrm{NB}$ and $\mathrm{BE}$ by one up to a maximum value macMaxCSMABackoffs and macMaxBE, respectively. Hence, the value of $\mathrm{NB}$ and $\mathrm{BE}$ depend on the number of $\mathrm{CCA}$ failures of a packet. Once the BE reaches macMaxBE, it remains at the value of macMaxBE until it is reset. If $\mathrm{NB}$ exceeds macMaxCSMABackoffs, then the packet is discarded due to the channel access failure. Otherwise the CSMA/CA algorithm generates a random number of complete backoff periods and repeat the process. Here, the variable macMaxCSMABackoffs represents the maximum number of times the CSMA/CA algorithm is required to backoff. If channel access is successful, the node starts transmitting packets and waits for acknowledgement (ACK). The reception of the corresponding ACK is interpreted as successful packet transmission. If the node fails to receive ACK due to collision or ACK timeout, the variable RT is increased by one up to macMaxFrameRetries. If RT is less than macMaxFrameRetries, the MAC sublayer initializes two variables $\mathrm{CW}=0, \mathrm{BE}=\operatorname{macMin} B E$ and follows the CSMA/CA mechanism to re-access the channel. Otherwise the packet is discarded due to the retry limit. Note that the default MAC parameters are macMinBE $=3, \operatorname{macMaxBE}=$ 5, macMaxCSMABackoffs $=4$, macMaxFrameRetries $=3$.

To account for the data processing time required at the MAC sublayer, two successive frames transmitted from a device are separated by at least an Inter-Frame Spacing (IFS) period; if the first transmission requires an acknowledgment, the separation between the ACK frame and the second transmission is at least an IFS period. Fig. 1 illustrates the IFS period of data frame with and without ACK. Note that the waiting time to receive ACK is in the range aTurnaroundTime (12 symbols) to aTurnaroundTime + aUnitBackoffPeriod $(12+20$ symbols $)$. The IFS period depends on the length of the transmitted data frames. See [1] for further details.

\section{Markov Chain Model}

In this section, we propose a generalized analytical model of the slotted CSMA/CA mechanism of beacon enabled IEEE 802.15.4 with retry limits for each packet transmission.

We consider a star network with a personal area network (PAN) coordinator, and $N$ nodes with beacon-enabled slotted CSMA/CA and ACK. All nodes contend to send data to the PAN coordinator, which is the data sink. Assume that the network generates an unsaturated traffic, which is a natural

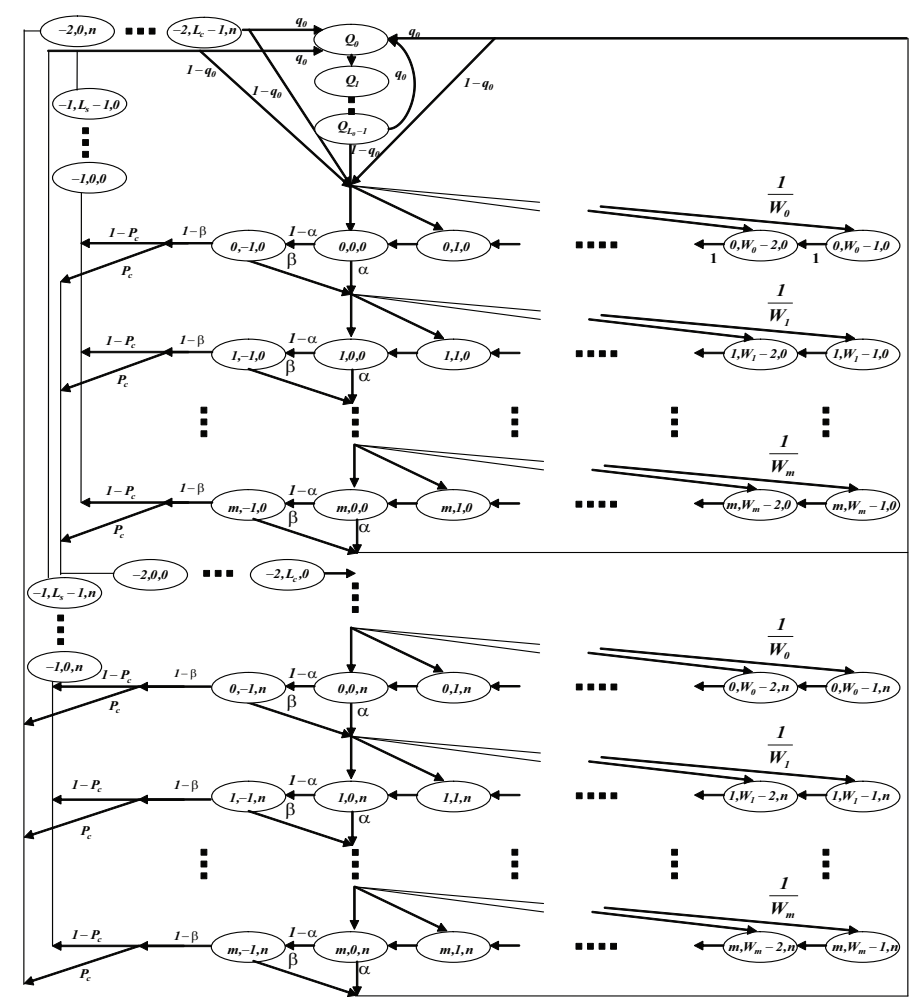

Fig. 2. Markov chain model for CSMA/CA algorithm for IEEE 802.15.4

scenario for many WSN applications. We study the behavior of a single node by using a Markov model.

Let $s(t), c(t)$ and $r(t)$ be the stochastic processes representing the backoff stage, the state of the backoff counter and the state of retransmission counter at time $t$, respectively, experienced by a node to transmit a packet as indicated in Fig. 2 . By assuming independent probability that nodes start sensing, the stationary probability $\tau$ that a node attempts a first carrier sensing in a randomly chosen time slot is constant and independent of other nodes, and the tuple $(s(t), c(t), r(t))$ is a three dimensional Markov chain. We denote the MAC parameters by $W_{0} \triangleq 2^{\operatorname{macMinBE}}, m_{0} \triangleq \operatorname{macMinBE}, m_{b} \triangleq \operatorname{macMaxBE}, m \triangleq$ macMaxCSMABackoffs, $n \triangleq$ macMaxFrameRetries. The states from $\left(i, W_{m}-1, j\right)$ to $\left(i, W_{0}-1, j\right)$ represent the backoff states. States $\left(Q_{0}, \ldots, Q_{L_{0}-1}\right)$ consider the idle state when the packet queue is empty and the node is waiting for new packet arrivals. Note that the idle states $\left(Q_{0}, \ldots, Q_{L_{0}-1}\right)$ take into account the unsaturated traffic condition. States $(i, 0, j)$ and $(i,-1, j)$ represent CCA1 and CCA2, respectively. Let $\alpha$ be the probability that CCA 1 is busy, $\beta$ the probability that CCA 2 is busy. Let $q_{0}$ the probability of going back to the idle state $Q_{0}$, and let $P_{c}$ be the probability that a transmitted packet encounters a collision. The states $(-1, k, j)$ and $(-2, k, j)$ model the successful transmission and packet collision, respectively. The state transition probabilities associated with the Markov chain of Fig. 2 are

$$
\begin{aligned}
& P(i, k, j \mid i, k+1, j)=1, \text { for } k \geq 0, \\
& P(i, k, j \mid i-1,0, j)=\frac{\alpha+(1-\alpha) \beta}{W_{i}}, \text { for } i \leq m,
\end{aligned}
$$




$$
\begin{aligned}
& P(0, k, j \mid i, 0, j-1)=\frac{(1-\alpha)(1-\beta) P_{c}}{W_{0}}, \text { for } j \leq n, \\
& P\left(Q_{0} \mid m, 0, j\right)=q_{0}(\alpha+(1-\alpha) \beta), \text { for } j<n, \\
& P\left(Q_{0} \mid i, 0, n\right)=q_{0}(1-\alpha)(1-\beta), \text { for } i<m, \\
& P\left(Q_{0} \mid m, 0, n\right)=q_{0}, \\
& P\left(0, k, 0 \mid Q_{0}\right)=\frac{1-q_{0}}{W_{0}}, \text { for } k \leq W_{0}-1 .
\end{aligned}
$$

Eq. (1) is the decrement of backoff counter, which happens with probability 1 . Eq. (2) represents the probability of finding busy channel either in CCA1 or CCA2 and of selecting a state uniformly the in the next backoff stage. Eq. (3) gives the unsuccessful transmission probability after finding an idle channel in both CCA1 and CCA2, and a node picks uniformly a state in the next retransmission stage. Eqs. (4) and (5) represent the probability of going back to the idle stage due to the channel access failure and retry limits, respectively. Eq. (6) is the probability of going back to the idle stage at backoff counter $m$ and retransmission stage $n$, as function of the traffic conditions $q_{0}$. Eq. (7) models the probability of going back to the first backoff stage from the idle stage. In the following, we use Eqs. (1)-(7) to compute the stationary distribution of the Markov chain.

Let $b_{i, k, j}=\lim _{t \rightarrow \infty} \operatorname{Pr}(s(t)=i, c(t)=k, r(t)=j), i \in$ $(-2, m), k \in\left(-1, \max \left(W_{i}-1, L_{s}-1, L_{c}-1\right)\right), j \in(0, n)$ be the stationary distribution of the Markov chain where $L_{s}, L_{c}$ are the time period for successful transmission and packet collision, respectively. Next, we derive the closed form expression for such distribution chain. Owing to the chain regularities and Eqs. (1)- (7), we have

$$
b_{i, k, j}=\frac{W_{i}-k}{W_{i}} b_{i, 0, j},
$$

where

$$
W_{i}=\left\{\begin{array}{l}
2^{i} W_{0} \quad i \leq m_{b}-m_{0} \\
2^{m_{b}-m_{0}} W_{0} \quad i>m_{b}-m_{0} .
\end{array}\right.
$$

From Eq. (2), for $i \leq m$ we obtain

$$
b_{i, 0, j}=(\alpha+(1-\alpha) \beta)^{i} b_{0,0, j} .
$$

From Eq. (3), $b_{0,0, j}$ is rewritten as follows

$$
\begin{aligned}
b_{0,0, j} & =(1-\alpha)(1-\beta) P_{c} \sum_{i=0}^{m} b_{i, 0, j-1} \\
& =\left((1-\alpha)(1-\beta) P_{c} \sum_{i=0}^{m}(\alpha+(1-\alpha) \beta)^{i}\right)^{j} b_{0,0,0} .
\end{aligned}
$$

By the normalization condition, we know that

$$
\begin{aligned}
& \sum_{i=0}^{m} \sum_{k=0}^{W_{i}-1} \sum_{j=0}^{n} b_{i, k, j}+\sum_{i=0}^{m} \sum_{j=0}^{n} b_{i,-1, j} \\
& +\sum_{j=0}^{n}\left(\sum_{k=0}^{L_{s}-1} b_{-1, k, j}+\sum_{k=0}^{L_{c}-1} b_{-2, k, j}\right)+\sum_{l=0}^{L_{0}-1} Q_{l}=1 .
\end{aligned}
$$

We next derive the expressions of each term in Eq. (11).
From Eqs. (8), (9), (10), we have

$$
\begin{aligned}
& \sum_{i=0}^{m} \sum_{k=0}^{W_{i}-1} \sum_{j=0}^{n} b_{i, k, j} \\
& =\sum_{i=0}^{m} \sum_{j=0}^{n} \frac{W_{i}+1}{2}(\alpha+(1-\alpha) \beta)^{i} b_{0,0, j} \\
& =\left\{\begin{array}{c}
\frac{b_{0,0,0}}{2}\left(\frac{1-(2 x)^{m+1}}{1-2 x} W_{0}+\frac{1-x^{m+1}}{1-x}\right) \frac{1-y^{n+1}}{1-y} \\
\text { if } m \leq m_{b}-m_{0} \\
\frac{b_{0,0,0}}{2}\left(\frac{1-(2 x)^{m_{b}-m_{0}+1}}{1-2 x} W_{0}+\frac{1-x^{m_{b}-m_{0}+1}}{1-x}+\right. \\
\left.\left(2^{m_{b}}+1\right) x^{m_{b}-m_{0}+1} \frac{1-x^{m-m_{b}+m_{0}}}{1-x}\right) \frac{1-y^{n+1}}{1-y} \\
\text { otherwise, }
\end{array}\right.
\end{aligned}
$$

where $x=\alpha+(1-\alpha) \beta$ and $y=P_{c}\left(1-x^{m+1}\right)$. Similarly,

$$
\begin{aligned}
\sum_{i=0}^{m} \sum_{j=0}^{n} b_{i,-1, j} & =\sum_{i=0}^{m} \sum_{j=0}^{n}(1-\alpha)(\alpha+(1-\alpha) \beta)^{i} b_{0,0, j} \\
& =(1-\alpha) \frac{1-x^{m+1}}{1-x} \frac{1-y^{n+1}}{1-y} b_{0,0,0},
\end{aligned}
$$

and

$$
\begin{aligned}
& \sum_{j=0}^{n}\left(\sum_{k=0}^{L_{s}-1} b_{-1, k, j}+\sum_{k=0}^{L_{c}-1} b_{-2, k, j}\right) \\
& \quad=\left(L_{s}\left(1-P_{c}\right)+L_{c} P_{c}\right)\left(1-x^{m+1}\right) \frac{1-y^{n+1}}{1-y} b_{0,0,0}
\end{aligned}
$$

By considering that the successful transmission and the failure events are due to the limited number of backoff stages $m$ and the retry limits $n$, the idle state probability is

$$
\begin{aligned}
Q_{0}= & q_{0} Q_{L_{0}-1}+q_{0}\left[\sum_{j=0}^{n}(\alpha+(1-\alpha) \beta) b_{m, 0, j}\right. \\
& \left.+\sum_{i=0}^{m} P_{c}(1-\beta) b_{i,-1, n}+\sum_{i=0}^{m} \sum_{j=0}^{n}\left(1-P_{c}\right)(1-\beta) b_{i,-1, j}\right] \\
= & \frac{q_{0}}{1-q_{0}}\left[\frac{x^{m+1}\left(1-y^{n+1}\right)}{1-y}+P_{c}\left(1-x^{m+1}\right) y^{n}\right. \\
& \left.+\left(1-P_{c}\right) \frac{\left(1-x^{m+1}\right)\left(1-y^{n+1}\right)}{1-y}\right] b_{0,0,0},
\end{aligned}
$$

where $L_{0}$ is the idle state length without generating packets and $\sum_{l=0}^{L_{0}-1} Q_{l}=L_{0} Q_{0}$. Note that Eqs. (12)-(15) give the state values $b_{i, k, j}$ as a function of $b_{0,0,0}$. By replacing Eqs. (12)-(15) in the normalization condition given by Eq. (11), we obtain the expression for $b_{0,0,0}$.

\section{ACCURATE ANALYSis}

In this section, we derive the accurate expressions of the reliability, delay and energy consumption offered by IEEE 802.15 .4 by using the Markov chain developed in the previous section.

\section{A. Reliability}

To derive the probability of successful packet reception, or reliability, we derive first the probability $\tau$ that a node attempts 
a first carrier sensing (CCA1) in a randomly chosen time slot is

$$
\tau=\sum_{i=0}^{m} \sum_{j=0}^{n} b_{i, 0, j}=\left(\frac{1-x^{m+1}}{1-x}\right)\left(\frac{1-y^{n+1}}{1-y}\right) b_{0,0,0} .
$$

The probability $\tau$ depends on the probability $P_{c}$ that a transmitted packet encounters a collision, the probability $\alpha$ that CCA 1 is busy, and the probability $\beta$ that CCA 2 is busy. We study these three probabilities next.

The term $P_{c}$ is the probability that at least one of the $N-1$ remaining nodes transmits in the same time slot. If all nodes transmit with probability $\tau, P_{c}$ is

$$
P_{c}=1-(1-\tau)^{N-1}
$$

where $N$ is the number of nodes. Similarly to [8], we derive the busy channel probabilities $\alpha$ and $\beta$ as follows. Since

$$
\alpha=\alpha_{1}+\alpha_{2}
$$

where $\alpha_{1}$ is the probability of finding channel busy during CCA1 due to data transmission, namely

$$
\alpha_{1}=L\left(1-(1-\tau)^{N-1}\right)(1-\alpha)(1-\beta),
$$

and $\alpha_{2}$ is the probability of finding the channel busy during CCA1 due to ACK transmission, which is

$\alpha_{2}=L_{\text {ack }} \frac{N \tau(1-\tau)^{N-1}}{1-(1-\tau)^{N}}\left(1-(1-\tau)^{N-1}\right)(1-\alpha)(1-\beta)$,

where $L_{\text {ack }}$ is the length of the ACK. Finally,

$$
\beta=\frac{1-(1-\tau)^{N-1}+N \tau(1-\tau)^{N-1}}{2-(1-\tau)^{N}+N \tau(1-\tau)^{N-1}} .
$$

The expressions of the carrier sensing probability $\tau$ and the busy channel probabilities $\alpha$ and $\beta$ form a system of non-linear equations that can be solved through a numerical method.

In slotted CSMA/CA, packets are discarded due to two reasons: (i) channel access failure (ii) retry limits. Channel access failure happens when a packet fails to obtain idle channel in two consecutive CCAs within $m+1$ backoffs. Furthermore, a packet is discarded if the transmission fails due to repeated collisions after $n+1$ attempts. Following the Markov model presented in Fig. 2, the probability that the packet is discarded due to channel access failure is

$$
P_{c f}=\sum_{j=0}^{n} x b_{m, 0, j}=\frac{x^{m+1}\left(1-y^{n+1}\right)}{1-y} .
$$

The probability of a packet being discarded due to retry limits is

$$
P_{c r}=\sum_{i=0}^{m} P_{c}(1-\beta) b_{i,-1, n}=y^{n+1} .
$$

Therefore, by using Eq. (19) and (20), the reliability is given by

$$
R=1-P_{c f}-P_{c r}
$$

\section{B. Delay}

The average delay for a successfully received packet is defined as the time interval from the instant the packet is at the head of its MAC queue and ready to be transmitted, until an ACK for such a packet is received. If a packet is dropped due to either the limited backoffs $m$ or the finite retry limit $n$, its delay is not included into the average delay.

Let $D_{j}$ be the event that a node sends a packet successfully at the $j$ th time. Then, from the Marov model, the random variables $\left(D_{j}-D_{j-1}\right)$ and $\left(D_{j+1}-D_{j}\right)$ are independent. Let $T_{h, i}$ be the random time needed to obtain two successful CCAs from the selected backoff counter value in backoff level $i$. Recalling from Section II, a node transmits the packet when the backoff counter is 0 and two successful CCAs occur. The transmission may be successful with probability $1-P_{c}$, or collide with probability $P_{c}$. The total delay $D$ to have a successful transmission within $n$ unsuccessful attempts is

$$
D=\sum_{j=0}^{n} \mathbb{1}\left(A_{j} \mid A_{t}\right) D_{j}
$$

where $D_{j}=L_{s}+j L_{c}+\sum_{h=0}^{j} T_{h}, T_{h}$ is the backoff stage delay, $L_{s}$ and $L_{c}$ are the time periods for successful packet transmission and collided packet transmission, respectively. The event $A_{j}$ denotes the occurrence of a successful packet transmission at time $j+1$ given $\mathrm{j}$ previous unsuccessful transmissions, whereas the event $A_{t}$ denotes the occurrence of a successful packet transmission within $n$ attempts. By knowing the time duration of ACK frame, ACK timeout, IFS, data packet length and header duration, we compute $L_{s}, L_{c}$ as

$$
\begin{aligned}
& L_{s}=L+t_{\mathrm{ack}}+L_{\mathrm{ack}}+I F S, \\
& L_{c}=L+t_{\mathrm{m}, \text { ack }},
\end{aligned}
$$

where $L$ is the total length of packet including overhead and payload, $t_{\text {ack }}$ is ACK waiting time, $L_{\text {ack }}$ is the length of ACK frame, IFS is Inter-Frame Spacing and $t_{\mathrm{m} \text {,ack }}$ is the timeout of ACK, see the details in Section II and [1]. We then have

$$
\begin{aligned}
\operatorname{Pr}\left(A_{j} \mid A_{t}\right) & =\frac{P_{c}^{j}\left(1-x^{m+1}\right)^{j}}{\sum_{k=0}^{n}\left(P_{c}\left(1-x^{m+1}\right)\right)^{k}}, \\
& =\frac{\left(1-P_{c}\left(1-x^{m+1}\right)\right) P_{c}^{j}\left(1-x^{m+1}\right)^{j}}{1-\left(P_{c}\left(1-x^{m+1}\right)\right)^{n+1}}
\end{aligned}
$$

where $P_{c}$ is the collision probability per sending attempt and $\left(1-x^{m+1}\right)$ is the probability of successful channel accessing within the maximum number of $m$ backoff stages. Note that the probability of the event $A_{j}$ is normalized by considering all the possible events of successful attempts $A_{t}$. Hence, the expected value of $D$ is

$$
\mathbb{E}[D]=\sum_{j=0}^{n} \operatorname{Pr}\left(A_{j} \mid A_{t}\right) \mathbb{E}\left[D_{j}\right],
$$

where $\mathbb{E}\left[D_{j}\right]=T_{s}+j T_{c}+\sum_{h=0}^{j} \mathbb{E}\left[T_{h}\right]$.

By following a similar approach as the one for the characterization of $D$, we see that the total backoff delay $T_{h}$ is modelled by

$$
T_{h}=\sum_{i=0}^{m} \mathbb{1}\left(B_{i} \mid B_{t}\right) T_{h, i}
$$


where

$$
T_{h, i}=2 T_{s c}+\sum_{k=1}^{i} T_{h, k}^{s c}+\sum_{k=0}^{i} T_{h, k}^{b},
$$

and where $2 T_{s c}$ is the successful sensing time, $\sum_{k=1}^{i} T_{h, k}^{s c}$ is the unsuccessful sensing time due to busy channel during CCA, and $\sum_{k=0}^{i} T_{h, k}^{b}$ is the backoff time. The event $B_{i}$ denotes the occurrence of a busy channel for $i$-th times, and then of idle channel at the $i+1$ th time. By considering all the possibilities of busy channel during two CCAs, the probability of $B_{i}$ is conditioned on the successful sensing event within $m$ attempts $B_{t}$, given that the node senses an idle channel in CCA. It follows that

$$
\operatorname{Pr}\left(B_{i} \mid B_{t}\right)=\frac{\sum_{k=1}^{2^{i}} C_{\alpha \beta}^{k}(i)}{\sum_{k=0}^{m} C_{\alpha \beta}(k)},
$$

where $C_{\alpha \beta}(i)$ gives all possibilities of choosing $i$ elements from a set of busy channel probabilities $\{\alpha,(1-\alpha) \beta\}$ and $C_{\alpha \beta}^{k}(i)$ is one of the elements in the set $C_{\alpha \beta}(i)$. Hence, the total number of combinations for $i$ elements is equal to $2^{i}$ and $C_{\alpha \beta}^{k}(i)$ returns one combination out of $2^{i}$. The expected backoff delay is

$$
\mathbb{E}\left[T_{h}\right]=\sum_{i=0}^{m} \operatorname{Pr}\left(B_{i} \mid B_{t}\right) \mathbb{E}\left[T_{h, i}\right] .
$$

Note that $\mathbb{E}\left[T_{h, i}\right]$ follows from Eq. (24). The unsuccessful sensing time $\sum_{k=1}^{i} T_{h, k}^{s c}$ in Eq. (24) is related to the picking of $i$ elements in the set $C_{\alpha \beta}(i)$. For instance, the combination $(\alpha, \alpha)$ returns the unsuccessful sensing delay $T_{s c}+T_{s c}$ and the combination $(\alpha,(1-\alpha) \beta)$ gives the unsuccessful sensing delay $T_{s c}+2 T_{s c}$. Furthermore, the backoff time $T_{h, k}^{b}$ of $k$ unsuccessful sensing tries is uniformly distributed in $\left[0, W_{k}-1\right]$. Hence, we can rewrite the expected backoff delay $\mathbb{E}\left[T_{h}\right]$ as

$$
\begin{aligned}
\mathbb{E}\left[T_{h}\right]= & 2 T_{s c}+\sum_{k=0}^{m} \operatorname{Pr}\left(B_{i} \mid B_{t}\right) \sum_{k=0}^{i} \frac{W_{k}-1}{2} S_{b} \\
& +\frac{T_{s c}}{\sum_{k=0}^{m} C_{\alpha \beta}(k)} \sum_{i=0}^{m} \sum_{k=1}^{2^{i}} C_{\alpha \beta}^{k}(i)\left(N_{\alpha}^{k}(i)+2 N_{\hat{\beta}}^{k}(i)\right),
\end{aligned}
$$

where $S_{b}$ is the time unit aUnitBackoffPeriod, and $N_{\alpha}^{k}(i)$, $N_{\hat{\beta}}^{k}(i)$ return the number of $\alpha$ and $(1-\alpha) \beta$ of the combination $C_{\alpha \beta}^{k}(i)$, respectively.

By a similar approach, the variance of the total delay is

$$
\sigma^{2}[D]=\sum_{j=0}^{n} \operatorname{Pr}\left(A_{j} \mid A_{t}\right)\left[\mathbb{E}\left[D_{j}^{2}\right]-(\mathbb{E}[D])^{2}\right] .
$$

\section{Energy Consumption}

By considering the Markov chain model given in Fig. 2, the average energy consumption is given as follow

$$
\begin{aligned}
E_{\mathrm{tot}}= & P_{i} \sum_{i=0}^{m} \sum_{k=1}^{W_{i}-1} \sum_{j=0}^{n} b_{i, k, j}+P_{s c} \sum_{i=0}^{m} \sum_{j=0}^{n}\left(b_{i, 0, j}+b_{i,-1, j}\right) \\
& +P_{t} \sum_{j=0}^{n} \sum_{k=0}^{L-1}\left(b_{-1, k, j}+b_{-2, k, j}\right)+P_{i} \sum_{j=0}^{n}\left(b_{-1, L, j}\right. \\
& \left.+b_{-2, L, j}\right)+\sum_{j=0}^{n} \sum_{k=L+1}^{L+L_{\text {ack }}+1}\left(P_{r} b_{-1, k, j}+P_{i} b_{-2, k, j}\right) \\
& +P_{s p} \sum_{l=0}^{L_{0}-1} Q_{l}
\end{aligned}
$$

where $P_{i}, P_{s c}, P_{t}, P_{r}$ and $P_{s p}$ are the average energy consumption in idle-listen, channel sensing, transmit, receiving, and sleep states, respectively. We assume that the radio is set in idle-listen state during the backoff stages and the timeout of ACK, $t_{\mathrm{m}, \text { ack }}=L_{\mathrm{ack}}+1$, in time units $S_{b}$. In Eq. (27), the first and second terms take into account the energy consumption during idle backoff state and channel sensing state, respectively. The third, fourth and fifth terms consider the energy consumption of packet transmission stage. The last term is the energy consumption of idle stage without packet generation. By substituting Eqs. (12)-(15) to Eq. (27), we obtain the average energy consumption in closed form.

\section{Approximated AnALysis}

In previous sections we presented a generalized Markov chain model of the CSMA/CA mechanism, and we gave the expressions of the reliability, delay for successful packet delivery, and energy consumption. These expressions are based on the nonlinear Eqs. (16)-(18), which must be solved through a numerical method. However, these expressions may be computationally demanding and inadequate for usage in sensor devices. For instance, a node may need to solve locally an optimization problem where the cost function is given by the energy (27), and the constraints are imposed by the reliability (21) and delay (23) expressions. We argue that simpler expressions for such an optimization problem are needed for an in-network solution [19].

In this section, we approximate the accurate model and analysis developed in Section III by simpler expressions. The key idea is that sensor nodes can easily estimate the busy channel probabilities $\alpha, \beta$ and the probability $\tau$. Therefore, we propose some approximated expressions where nodes exploit local measurements to evaluate reliability, delay, and energy consumption, rather than solving nonlinear equations. In the following, we give these approximations. Recall that we defined $x=\alpha+(1-\alpha) \beta$ and $y=P_{c}\left(1-x^{m+1}\right)$.

\section{A. Reliability}

To approximate the reliability expression of Eq. (21), we first consider the carrier sensing probability $\tau$ of Eq. (16), where the state $b_{0,0,0}$ follows from the normalization condition in Eq. (11). Given $z \geq 0$, note that

$$
\frac{1-z^{m+1}}{1-z} \approx 1+z \quad \text { if } \quad z \ll 1
$$

By using this approximation, Eq. (12) is approximated as

$$
\sum_{i=0}^{m} \sum_{k=0}^{W_{i}-1} \sum_{j=0}^{n} b_{i, k, j} \approx \frac{b_{0,0,0}}{2}\left[(1+2 x) W_{0}+1+x\right](1+y)
$$

Similarly, Eq. (13) is approximated by

$$
\sum_{i=0}^{m} \sum_{j=0}^{n} b_{i,-1, j} \approx b_{0,0,0}(1-\alpha)(1+x)(1+y)
$$

and Eq. (14) is approximated by

$$
\begin{gathered}
\sum_{j=0}^{n}\left(\sum_{k=0}^{L_{s}-1} b_{-1, k, j}+\sum_{k=0}^{L_{c}-1} b_{-2, k, j}\right) \\
\approx b_{0,0,0} L_{s}\left(1-x^{m+1}\right)(1+y),
\end{gathered}
$$


where we assume that the successful packet service time is equal to the packet collision time, namely $L_{s}=L_{c}$. Finally, let $K_{0}=L_{0} q_{0} /\left(1-q_{0}\right)$, then the approximate idle stage of Eq. (15) is

$$
\sum_{l=0}^{L_{0}-1} Q_{l} \approx b_{0,0,0} K_{0}\left[1+y+P_{c}\left(1-x^{m+1}\right)\left(y^{n}-y-1\right)\right] \text {. }
$$

By summing together Eqs. (29)-(32), the approximated state probability $\widetilde{b}_{0,0,0}$ is

$$
\begin{aligned}
\widetilde{b}_{0,0,0} \approx & {\left[\frac{W_{0}}{2}(1+2 x)(1+y)+L_{s}\left(1-x^{2}\right)(1+y)\right.} \\
& \left.+K_{0}\left(\left(P_{c}\left(1-x^{2}\right)\right)^{2}\left(\left(P_{c}\left(1-x^{2}\right)\right)^{n-1}+1\right)+1\right)\right]^{-1}
\end{aligned}
$$

where we neglect the term in $\mathrm{Eq}(30)$ and use $1-x^{m+1} \approx$ $1-x^{2}$.

In a similar way, the carrier sensing probability given by Eq. (16) is approximated as $\widetilde{\tau}=(1+x)(1+y) \widetilde{b}_{0,0,0}$. Hence, the approximated reliability is

$$
\widetilde{R}=1-x^{m+1}(1+\widetilde{y})-\widetilde{y}^{n+1},
$$

where $\widetilde{y}=\left(1-(1-\widetilde{\tau})^{N-1}\right)\left(1-x^{2}\right) . \widetilde{R}$ is a function of the busy channel probability $\alpha, \beta$, the collision probability $P_{c}$ and the MAC parameters $m_{0}, m_{b}, m, n$.

\section{B. Delay}

The average delay given by Eq. (23) is approximated as

$$
\mathbb{E}[\widetilde{D}]=\mathbf{P}^{T} \mathbf{D}
$$

where $\mathbf{P}=\left[\operatorname{Pr}\left(A_{0} \mid A_{t}\right) \cdots \operatorname{Pr}\left(A_{n} \mid A_{t}\right)\right]^{T} \in \mathbb{R}^{(n+1) \times 1}, \mathbf{D}=$ $\left[d_{0} \cdots d_{n}\right]^{T} \in \mathbb{R}^{(n+1) \times 1}, d_{j}=T_{s}+j T_{c}+(j+1) \mathbb{E}[\widetilde{T}]$, and where $\operatorname{Pr}\left(A_{j} \mid A_{t}\right)$ is given by Eq. (22). $\mathbb{E}[\widetilde{T}]$ is the approximation of the average backoff period:

$$
\begin{aligned}
\mathbb{E}[\widetilde{T}] & =2 T_{s c}+\sum_{i=0}^{m} \widetilde{P}\left(B_{i} \mid B_{t}\right) \sum_{k=0}^{i}\left(\frac{W_{0} 2^{k}-1}{2} S_{b}+2 T_{s c} k\right) \\
& =2 S_{b}\left(1+\widetilde{\mathbf{P}}^{T} \mathbf{T}\right)
\end{aligned}
$$

where $\widetilde{\mathbf{P}}=\left[\widetilde{P}\left(B_{0} \mid B_{t}\right) \cdots \widetilde{P}\left(B_{m} \mid B_{t}\right)\right]^{T} \in \mathbb{R}^{(m+1) \times 1}, \mathbf{T}=$ $\left[t_{0} \cdots t_{m}\right]^{T} \in \mathbb{R}^{(m+1) \times 1}, \widetilde{P}\left(B_{i} \mid B_{t}\right)$ is given by Eq. (36) and $t_{i}=\left[\left(2^{i+1}-1\right) W_{0}+3 i-1\right] / 4$. The approximation considers the worst case, i.e., a failure of the second sensing (CCA2), which implies that $T_{s c}=S_{b}$ and that each sensing failure takes $2 T_{s c}$. Under these assumptions, the probability of the event $B_{i}$ in Eq. (25) is approximated by

$$
\widetilde{P}\left(B_{i} \mid B_{t}\right)=\frac{\max (\alpha,(1-\alpha) \beta)^{i}}{\sum_{k=0}^{m} \max (\alpha,(1-\alpha) \beta)^{k}},
$$

where we did a further approximation by not considering all the possibilities of busy channel during two CCAs.

Now, we are in the position to give an approximation of the discrete probability distribution function of the delay. A probability generation function approach can be used to compute the discrete probability distribution of the delay. However, such an approach is computationally quite expensive. For analysis and optimization, some continuous wellknown distributions are used to approximate the simulation results. The approximated distribution is obtained by using a moment matching approach. Namely, the discrete probability distribution function of the delay is approximated by known distributions whose average and variance is matched to the actual average and variance of the delay. More specifically, let $D_{a}$ be an approximating delay distribution having average $\mu_{D_{a}}$ and variance $\sigma_{D_{a}}^{2}$. Then, we impose that $\mu_{D_{a}}$ and $\sigma_{D_{a}}^{2}$ are given by Eqs. (23) and (26), respectively. Typical distribution for $D_{a}$ should be one-sided, as the Exponential, Log-normal, Poisson, and Chi-square ones, since the delay is positively distributed. In Section VI, we evaluate the accuracy of the approximated probability distribution function of the delay as given by these one-sided distributions.

\section{Energy Consumption}

Finally, we propose an approximation of the average energy consumption. From Eq. (12), the average energy consumption of the backoff stage is

$$
\begin{aligned}
& P_{i} \sum_{i=0}^{m} \sum_{k=1}^{W_{i}-1} \sum_{j=0}^{n} b_{i, k, j} \\
& \quad=\frac{P_{i} \tau}{2}\left(\frac{(1-x)\left(1-(2 x)^{m+1}\right)}{(1-2 x)\left(1-x^{m+1}\right)} W_{0}-1\right)
\end{aligned}
$$

where we assume that the carrier sensing probability $\tau$ is measured by the node, i.e., it is not computed analytically.

By putting together Eqs. (12), (13) and (16), the average energy consumption of the sensing state is

$$
P_{s c} \sum_{i=0}^{m} \sum_{j=0}^{n}\left(b_{i, 0, j}+b_{i,-1, j}\right)=P_{s c}(2-\alpha) \tau .
$$

Similarly, by substituting Eq. (14) and Eq. (16), the average energy consumption for packet transmission including both successful transmission and packet collision is

$$
\begin{aligned}
P_{t} & \sum_{j=0}^{n} \sum_{k=0}^{L-1}\left(b_{-1, k, j}+b_{-2, k, j}\right)+P_{i} \sum_{j=0}^{n}\left(b_{-1, L, j}+b_{-2, L, j}\right) \\
& +\sum_{j=0}^{n} \sum_{k=L+1}^{L+L_{\text {ack }}+1}\left(P_{r} b_{-1, k, j}+P_{i} b_{-2, k, j}\right) \\
& =(1-\alpha)(1-\beta) \tau\left(P_{t} L+P_{i}+L_{\text {ack }}\left(P_{r}\left(1-P_{c}\right)+P_{i} P_{c}\right)\right) .
\end{aligned}
$$

We assume that the energy consumption at sleeping state is negligible, namely $P_{s p} \approx 0$. By summing up Eqs. (37), (38) and (39), the approximated average energy consumption is

$$
\begin{aligned}
\widetilde{E}_{\text {tot }}= & \frac{P_{i} \tau}{2}\left(\frac{(1-x)\left(1-(2 x)^{m+1}\right)}{(1-2 x)\left(1-x^{m+1}\right)} W_{0}-1\right)+P_{s c}(2-\alpha) \tau \\
& +(1-\alpha)(1-\beta) \tau\left(P_{t} L+P_{i}+L_{\text {ack }}\left(P_{r}\left(1-P_{c}\right)\right.\right. \\
& \left.\left.+P_{i} P_{c}\right)\right) .
\end{aligned}
$$

\section{Model Validation And Performance Analysis}

Here we present extensive Monte Carlo simulations of slotted IEEE 802.15.4 to validate our accurate and approximated expressions of the reliability, delay and energy consumption. The simulations are based on the specifications of the IEEE 802.15.4 [1] with several values of the traffic condition and MAC parameters. A performance analysis is also conducted. We investigate the effects of MAC parameters $m_{0}, m_{b}, m, n$ on the performance metrics. Details follow in the sequel. 


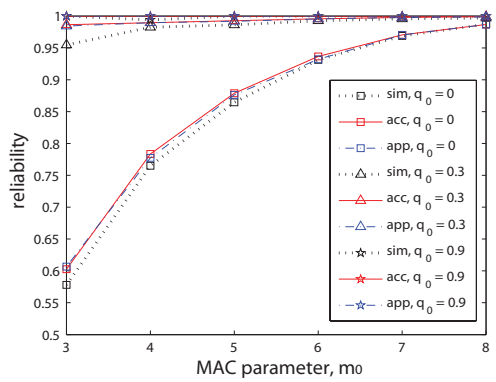

(a) $m_{0}=3, \ldots, 8, m_{b}=8, m=4, n=3$,

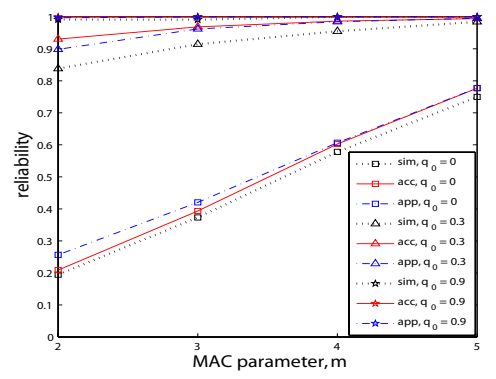

(b) $m=2, \ldots, 5, m_{0}=3, m_{b}=8, n=3$,

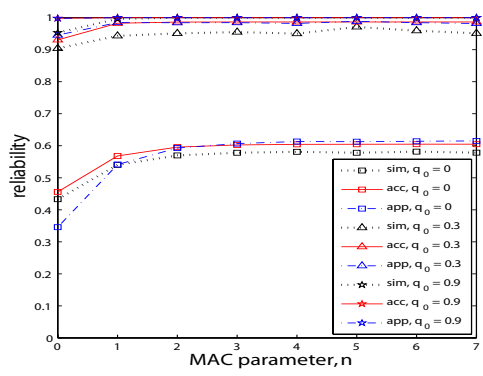

(c) $n=0, \ldots, 7, m_{0}=3, m_{b}=8, m=4$

Fig. 3. Reliability as a function of the traffic conditions $q_{0}=0,0.3,0.9$, and MAC parameters $m_{0}=3, \ldots, 8, m_{b}=8, m=2, \ldots, 5, n=0, \ldots, 7$. The length of the packet is $L=7$ and the number of nodes is $N=10$.

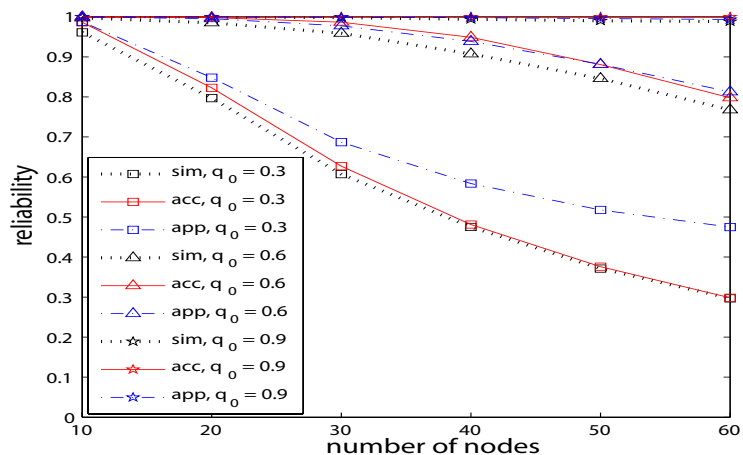

Fig. 4. Reliability as a function of traffic condition $q_{0}=0.3,0.6,0.9$ with a given MAC parameters $\left(m_{0}=3, m_{b}=8, m=4, n=3\right)$ and packet length $L=7$.

\section{A. Reliability Validation}

Fig. 4 illustrates the reliability as obtained by Monte Carlo simulations and the accurate expression Eq. (21) and approximated one Eq. (33) as a function of the traffic $q_{0}=0.3,0.6,0.9$ with a length of the packets $L=7$, and MAC parameters $m_{0}=3, m_{b}=8, m=4, n=3$. The accurate analytical model and approximated model match the simulation results quite well under low traffic condition $q_{0}=0.6,0.9$. However, the approximated expression shows a weak matching for the high traffic $q_{0}=0.3$ and large number of nodes $N \geq 30$. The reason is that the approximation given by Eq. (28) holds if $x \ll 1$, but $x$ increases as the traffic and the number of nodes increases.

Fig. 3 shows the reliability as obtained by Monte Carlo simulations, the accurate and approximated expressions as a function of the traffic conditions $q_{0}=0,0.3,0.9$ with a given number of nodes $N=10$ and different MAC parameters $m_{0}, m, n$. The accurate and approximated expressions match quite well the simulation results. The expressions are closer to simulation results under unsaturated traffic condition $q_{0}=0.3,0.9$ than saturated traffic condition $q_{0}=0$. The reliability approaches 1 under very low traffic regime $q_{0}=0.9$. In Figs. 3(a), 3(b), the reliability increases as MAC parameters $m_{0}, m$ increase, respectively. In Fig. 3(c), it is interesting to observe that the reliability does not improve as the retry limits $n$ increases for high traffic conditions $q_{0}=0$. Notice that the reliability saturates to 0.6 if $n \geq 2$. Hence, the retransmissions

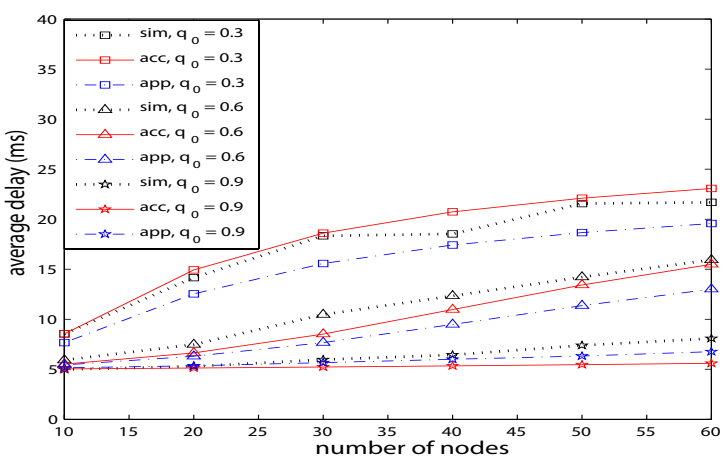

Fig. 5. Average delay as a function of traffic condition $q_{0}=0.3,0.6,0.9$ with a given MAC parameters $\left(m_{0}=3, m_{b}=8, m=4, n=3\right)$ and packet length $L=7$.

are necessary but not sufficient for high reliability under high traffic conditions.

\section{B. Delay Validation}

In Fig. 5 we report the average delay as obtained by Monte Carlo simulations, the accurate expression given by Eq. (23), and approximated one given by Eq. (34). The average delay is reported as a function of the traffic $q_{0}=0.3,0.6,0.9$, with a length of the packet $L=7$ and MAC parameters $m_{0}=3, m_{b}=8, m=4, n=3$. Similarly to the reliability, both the accurate and approximated expressions predict well the simulation results under low traffic condition $q_{0}=0.6,0.9$, whereas the approximation becomes less accurate for high traffic $q_{0}=0.3$ and large number of nodes $N \geq 30$.

Fig. 6 shows the average delay as a function of different traffic conditions $q_{0}=0.3,0.6,0.9$ with a given number of nodes $N=10$ and different MAC parameters $m_{0}, m, n$. Both the accurate and approximated expressions match well the simulation results, but the approximated model does not predict well the simulation results under high traffic condition $q_{0}=0.3$ due to the approximation given by Eq. (28). Observe that the average delay increases as traffic condition increases due to high busy channel probability and collision probability. Fig. 6(a) shows that the average delay increases exponentially as $m_{0}$ increases. Hence, we conclude that $m_{0}$ is the key parameter in terms of average delay with respect to $m$ and $n$. 


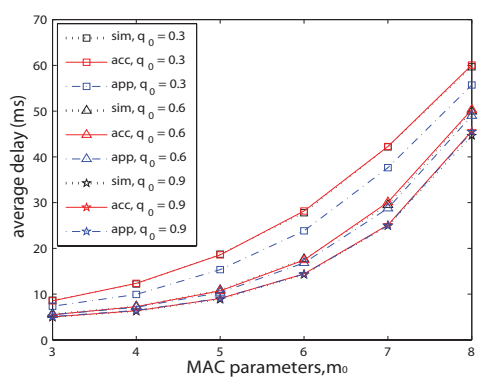

(a) $m_{0}=3, \ldots, 8, m_{b}=8, m=4, n=3$,

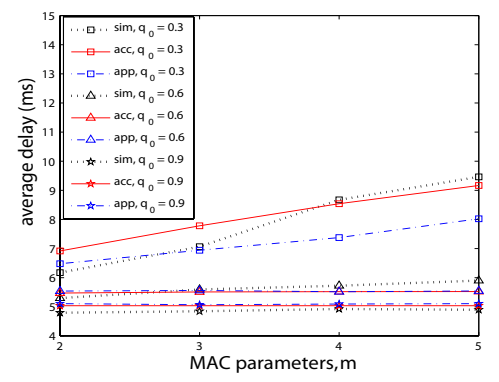

(b) $m=2, \ldots, 5, m_{0}=3, m_{b}=8, n=3$,

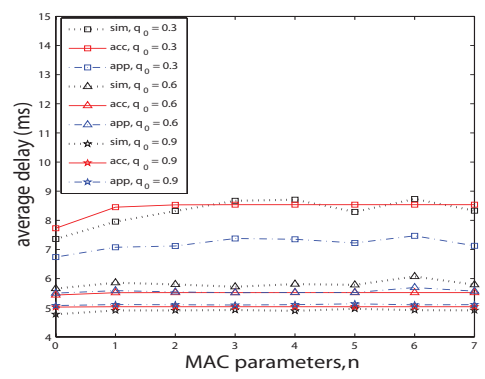

(c) $n=0, \ldots, 7, m_{0}=3, m_{b}=8, m=4$

Fig. 6. Average delay as a function of the traffic conditions $q_{0}=0.3,0.6,0.9$ and MAC parameters $m_{0}=3, \ldots, 8, m_{b}=8, m=2, \ldots, 5, n=0, \ldots, 7$. The length of the packet is $L=7$ and the number of nodes is $N=10$.

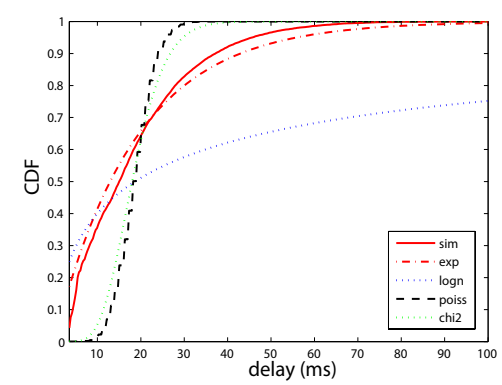

(a) $m_{0}=3, m_{b}=5, q_{0}=0, P_{c}=0.775$

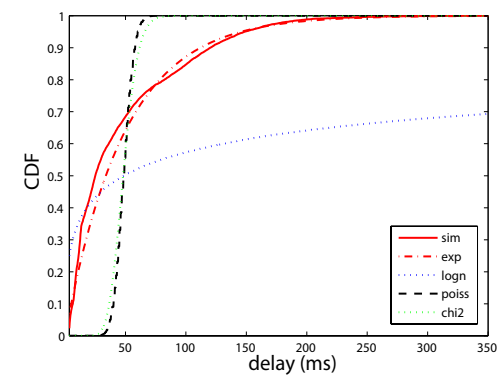

(d) $m_{0}=5, m_{b}=8, q_{0}=0, P_{c}=0.2766$

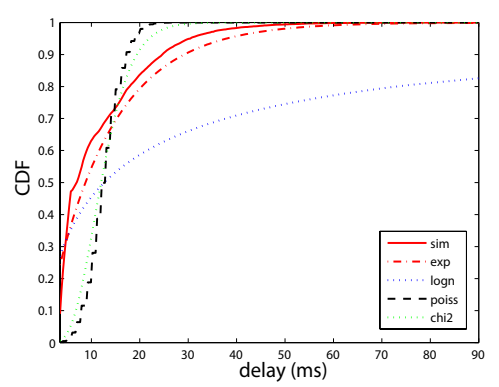

(b) $m_{0}=3, m_{b}=5, q_{0}=0.3, P_{c}=0.22$

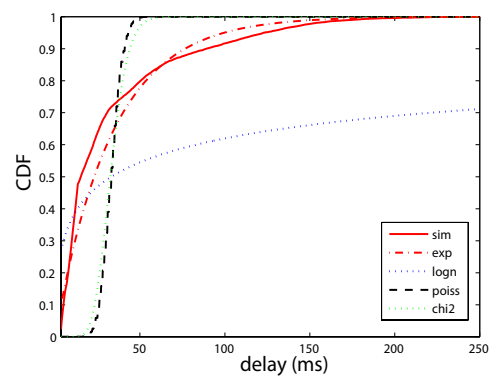

(e) $m_{0}=5, m_{b}=8, q_{0}=0.3, P_{c}=0.1347$

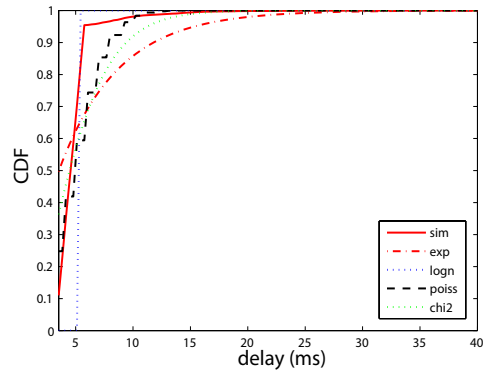

(c) $m_{0}=3, m_{b}=5, q_{0}=0.9, P_{c}=0.0044$

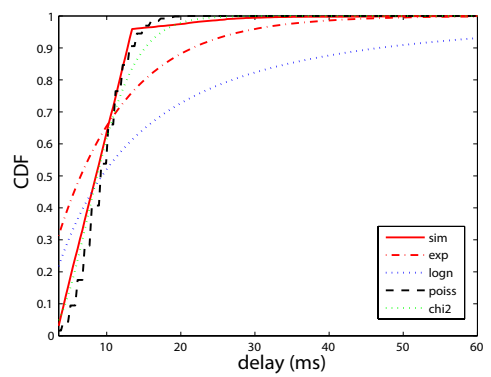

(f) $m_{0}=5, m_{b}=8, q_{0}=0.9, P_{c}=0.0044$

Fig. 7. Cumulative distribution function of the delay for successfully received packets as a function of the traffic conditions $q_{0}=0,0.3,0.9$ and different MAC parameters $m_{0}=3,5, m_{b}=5,8, m=4, n=3$. The length of the packet is $L=7$ and the number of nodes is $N=20$. The Exponential, Log-normal, Poisson, and Chi-square distribution are used for moment matching.

We check the validity of the delay distribution by using the approximation given by the moment matching approach described in Section V-B. Fig. 7 shows the cumulative distribution function (CDF) of packet delay as obtained by Monte Carlo simulations and the approximated distribution as a function of different parameters $m_{0}=3,5, m_{b}=5,8$, $m=4, n=3$, the packet length $L=7$, the number of nodes $N=20$ and different traffic conditions $q_{0}=0,0.3,0.9$. The moment matching has been obtained by using the Exponential, Log-normal, Poisson, and Chi-square distributions. In Figs. 7(a), 7(b), 7(d), 7(e), we see that the Exponential distribution predicts well the CDF for high collision probabilities. By contrast, in Figs. 7(c), 7(f), we observe that the Log-normal or Poisson distributions provide a good approximation for low collision probability. In addition, the Exponential distribution provides us with a fair approximation except for cases of very low collision probabilities. For these probabilities, the delay distribution is more similar to a deterministic distribution (see
Figs. 7(c), 7(f)). By comparing Figs. 7(a), 7(b) to 7(d), 7(e), we observe that a larger MAC parameter gives longer tails. From Figs. 7(c) and 7(f), we conclude that a good approximation of the distribution depends on both MAC parameters and collision probability.

To validate the accuracy of the approximated distributions by using a moment matching with the Exponential, Lognormal, Poisson, Chi-square distributions, the correlation coefficients $\rho^{2}$ between the simulation results and approximated distribution has been evaluated. Recall that the closer $\rho^{2}$ to 1 , the better the approximation. In the following, we validate the dependence between collision probability and correlation coefficient of the approximated distributions.

Figs. 8 show the relation of the correlation coefficient $\rho^{2}$ between the simulation results and the approximated distribution over different collision probabilities as a function of the different traffic condition $q_{0}=0.6,0.9$ and parameters $m_{0}=3,5, m_{b}=5,8, m=4, n=3$, the length of packet 


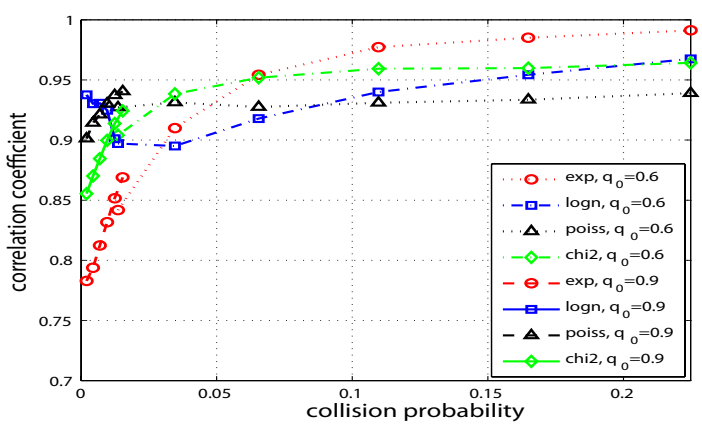

(a) $m_{0}=3, m_{b}=5, L=7, q_{0}=0.6,0.9$

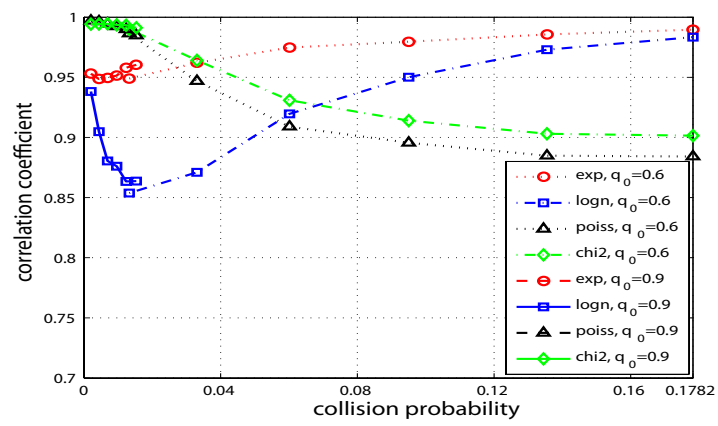

(c) $m_{0}=5, m_{b}=8, L=7, q_{0}=0.6,0.9$

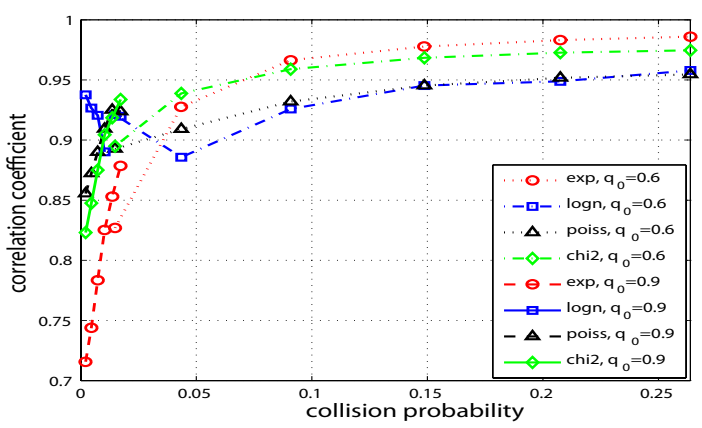

(b) $m_{0}=3, m_{b}=5, L=14, q_{0}=0.6,0.9$

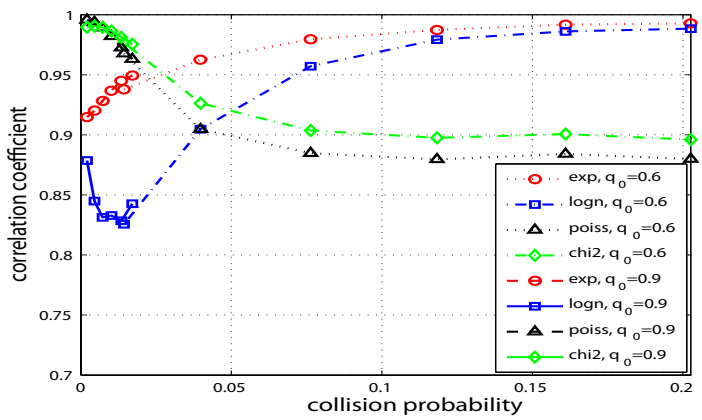

(d) $m_{0}=5, m_{b}=8, L=14, q_{0}=0.6,0.9$

Fig. 8. Correlation coefficients of approximated delay cumulative distribution function including Exponential, Log-normal, Poisson, Chi-square distributions as a function of different parameters $m_{0}=3,5, m_{b}=5,8, m=4, n=3$, the length of packet $L=7,14$ and the number of node $N=10,20,30,40,50,60$.

$L=7,14$ and the different number of nodes $N$. Hence, six different correlation coefficients and collision probabilities are displayed for a given traffic condition. From Fig. 8, we observe that the correlation coefficient depends mainly on collision probability $P_{c}$. In Fig. 8(a) and 8(b), the correlation coefficient is reported for a given set of MAC parameter $\left(m_{0}=3\right.$, $\left.m_{b}=5\right)$ and similar collision probabilities as those of Fig. 8. Observe that the correlation coefficient varies smoothly over different collision probabilities (see Figs. 8(c) and 8(d)). Therefore, we conclude that if the BEB mechanism does not have a strict limitation on the maximum value of the backoff exponent $m_{b}$ (as in IEEE 802.11), then the delay distribution is mainly dependent on collision probability (see Figs. 8(c) and $8(\mathrm{~d}))$. Otherwise, if MAC parameters $\left(m_{0}, m_{b}, m, n\right)$ have strict limitations as currently done in IEEE 802.15.4, then the delay distribution depends on both MAC parameters and collision probability.

Fig. 8 shows a good matching between the CDF of simulation results and the approximated distributions. Notice that the best correlation coefficient for the MAC parameters $m_{0}=5$ and $m_{b}=8$ is very close to 1 . The Exponential distribution gives the better match with the simulation results for $P_{c}>0.1$. In [14], the delay distribution of IEEE 802.11 matches well with a Log-normal distribution for almost all cases. However, the Log-normal distribution does not match well the simulation results for IEEE 802.15.4 as the collision probability increases. The reason is that the delay distribution for IEEE 802.15.4 does not have long tails compared to IEEE 802.11, because the MAC parameters have a strict limitation. Hence, for given MAC parameters, from our results we can

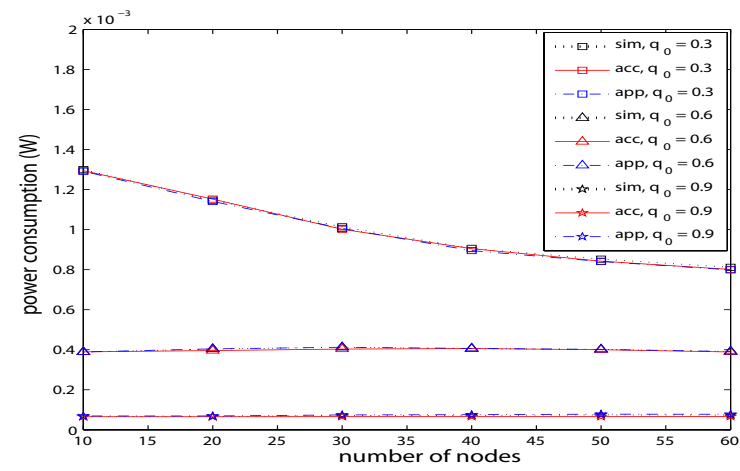

Fig. 9. Average energy consumption as a function of traffic condition $q_{0}=$ $0.3,0.6,0.9$ with MAC parameters $\left(m_{0}=3, m_{b}=8, m=4, n=3\right)$ and a given length of packet $L=7$.

choose the best approximated distribution out of Exponential, Log-normal, Poisson, Chi-square distributions by measuring collision probability.

\section{Energy Consumption Validation}

In Fig. 9 we reported the average energy consumption as achieved by Monte Carlo simulations, for the accurate expression given by Eq. (27) and the approximated expression given by Eq. (40). The curves depend on the traffic $q_{0}=0.3,0.6,0.9$ with a length of the packet $L=7$, and MAC parameters $m_{0}=3, m_{b}=8, m=4, n=3$. We observe that both the accurate and approximated expressions predict well the simulation results under different traffic conditions.

Fig. 10 shows the energy consumption as a function of different traffic conditions $q_{0}=0,0.3,0.9$ with a given number of 


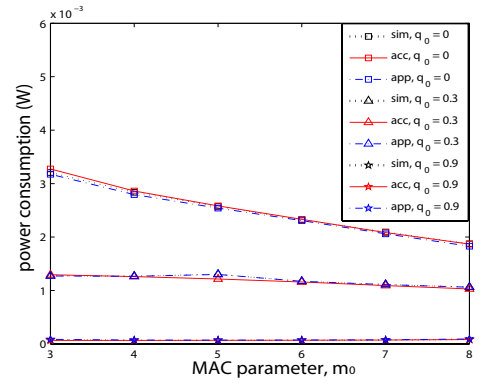

(a) $m_{0}=3, \ldots, 8, m_{b}=8, m=4, n=3$

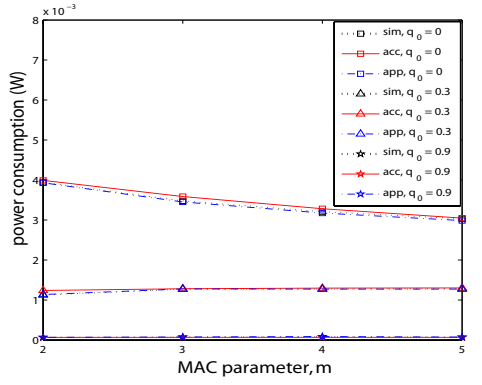

(b) $m=2, \ldots, 5, m_{0}=3, m_{b}=8, n=3$,

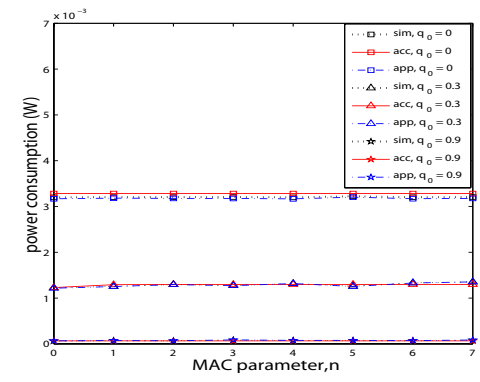

(c) $n=0, \ldots, 7, m_{0}=3, m_{b}=8, m=4$

Fig. 10. Average energy consumption as a function of the traffic conditions $q_{0}=0.3,0.6,0.9$ and MAC parameters $m_{0}=3, \ldots, 8, m_{b}=8, m=2, \ldots, 5$, $n=0, \ldots, 7$. The length of the packet is $L=7$ and the number of nodes is $N=10$.

nodes $N=10$ and different MAC parameters $m_{0}, m, n$. The accurate analytical model and approximated model match well the simulation results for all traffic conditions. It is interesting to observe that the energy consumption decreases as the MAC parameters $m_{0}, m, n$ increase under a high traffic condition $q_{0}=0$. In essence, as the MAC parameters increase, the node may stay more time in idle backoff stage than transmit or receiving mode i.e., $P_{r}>P_{i}>P_{s p}$ and $P_{t}>P_{i}>P_{s p}$. We observe that the energy consumption increases as MAC parameters $\left(m_{0}, m, n\right)$ increase under low traffic condition $q_{0}=0.3,0.9$. Since the node needs to stay more time in idle sleep stage without packet generation under low traffic condition $q_{0}=0.3,0.9$, the main component of average energy consumption is the idle backoff time rather than transmit or receiving energy consumption. It is interesting to observe that the energy consumption has a weaker dependence on the retry limits $n$ than the other MAC parameters $m_{0}, m$.

\section{CONCLUSIONS}

In this paper, we presented a generalized approach to analyze performance of the slotted CSMA/CA mechanism in the IEEE 802.15.4 standard. The approach is based on a Markov chain that considers retry limits, the acknowledgement mechanism, and unsaturated traffic, which are important components of most wireless sensor network applications. We derived the reliability, delay and energy consumption expressions offered by the slotted IEEE 802.15.4 standard by both an accurate and computationally demanding approach, and an approximate and simple approach. We showed that the approximated analysis is effective for low traffic. Furthermore, unlike 802.11, we observed that the delay distribution of IEEE 802.15.4 depends mainly on the MAC parameters and the collision probability. In addition, we analyzed the impact these parameters on the reliability, delay and energy consumption.

Future investigations include the use of the aforementioned achievements to the systematic design of optimized IEEE 802.15.4 MAC based on specific application requirements.

\section{REFERENCES}

[1] IEEE Std 802.15.4-2996, September, Part 15.4: Wireless Medium Access Control (MAC) and Physical Layer (PHY) Specifications for Low-Rate Wireless Personal Area Networks (WPANs), IEEE, 2006. [Online]. Available: http://www.ieee802.org/15
[2] G. Lu, B. Krishnamachari, and C. Raghavendra, "Performance evaluation of the IEEE 802.15.4 MAC for low-rate low-power wireless networks," in Proc. of IEEE IPCCC, 2004.

[3] J. Zheng and M. L. Lee, "A comprehensive performance study of IEEE 802.15.4," in IEEE Press Book, 2004.

[4] A. Koubaa, M. Alves, and E. Tovar, "A comprehensive simulation study of slotted CSMA/CA for IEEE 802.15.4 wireless sensor networks," in IEEE International Workshop on Factory Communication Systems, Jun 2006, pp. 183-192.

[5] S. Pollin, M. Ergen, S. C. Ergen, B. Bougard, L. V. D. Perre, F. Catthoor, I. Moerman, A. Bahai, and P. Varaiya, "Performance analysis of slotted carrier sense IEEE 802.15.4 medium access layer," in Proc. of IEEE GLOBECOM, 2006, pp. 1-6.

[6] J. Mišió, S. Shaf, and V. Mišió, "Performance of a beacon enabled IEEE 802.15.4 cluster with downlink and uplink traffic," in IEEE Trans. Parallel and Distributed Systems, 2006, pp. 361-376.

[7] P. K. Sahoo and J. P. Sheu, "Modeling IEEE 802.15.4 based wireless sensor network with packet retry limits," in PE-WASUN, 2008, pp. 6370.

[8] S. Pollin, M. Ergen, S. C. Ergen, B. Bougard, F. Catthoor, A. Bahai, and P. Varaiya, "Performance analysis of slotted carrier sense IEEE 802.15.4 acknowledged uplink transmissions," in Proc. of IEEE WCNC, 2008, pp. $1559-1564$.

[9] G. Bianchi, "Performance analysis of the IEEE 802.11 distributed cordination function," in IEEE Journal on Selected Areas in Communications, vol. 18, March 2000.

[10] IEEE Std 802.11 Wireless LAN Medium Access Control (MAC) and Physical Layer (PHY) Specifications, IEEE, 1999. [Online]. Available: http://www.ieee802.org/11

[11] P. Chatzimisios, A. C. Boucouvalas, and V. Vitsas, "IEEE 802.11 packet delay a finite retry limit analysis," in Proc. of IEEE GLOBECOM, 2003, pp. 950-954.

[12] P. Chatzimisios, V. Vitsas, and A. C. Boucouvalas, "Throughput and delay analysis of IEEE 802.11 protocol," in Proc. of IEEE IWNA, 2002, pp. $168-174$.

[13] Z. Hadzi-Velkov and B. Spasenovski, "Saturation throughput-delay analysis of IEEE 802.11 in fading channel," in Proc. of IEEE ICC, 2003.

[14] H. Zhai, Y. Kwon, and Y. Fang, "Performance analysis of IEEE 802.11 MAC protocols in wireless LANs: Research articles," in Wirel. Commun. Mob. Comput., 2004, pp. 917-931.

[15] O. Tickioo and B. Sikdar, "Queueing analysis and delay mitigation in IEEE 802.11 random access MAC based wireless networks," in Proc. of IEEE INFOCOM, 2004.

[16] H. Wu, Y. Peng, K. Long, S. Cheng, and J. Ma, "Performance of reliable transport protocol over IEEE 802.11 wireless LAN: Analysis and enhancement," in Proc. of IEEE INFOCOM, 2002, pp. 599-607.

[17] F. Cali, M. Conti, and E. Gregori, "Dynamic tuning of the IEEE 802.11 protocol to achieve a theoretical throughput limit," in IEEE Trans. Networking, Dec 2000, pp. 785-790.

[18] P. Raptis, A. Banchs, and K. Paparrizos, "A simple and effective delay distribution analysis for IEEE 802.11," in Proc. of IEEE PIMRC, 2006, pp. $1-5$.

[19] A. Giridhar and P. R. Kumar, "Toward a Theory of In-network Computation in Wireless Sensor Networks," IEEE Communication Magazine, pp. 97-107, April 2006. 